\title{
Incidence, Risk Factors, and Prediction of Myocardial Infarction and Stroke in Farmers: A Korean Nationwide Population-based Study
}

\author{
Solam Lee', Hunju Lee', Hye Sim Kim², Sang Baek Koh' \\ ${ }^{1}$ Department of Preventive Medicine, Yonsei University Wonju College of Medicine, Wonju, Korea; ${ }^{2}$ Center of Biomedical Data Science, Yonsei \\ University Wonju College of Medicine, Wonju, Korea
}

Objectives: This study was conducted to determine the incidence and risk factors of myocardial infarction (MI) and stroke in farmers compared to the general population and to establish 5-year prediction models.

Methods: The farmer cohort and the control cohort were generated using the customized database of the National Health Insurance Service of Korea database and the National Sample Cohort, respectively. The participants were followed from the day of the index general health examination until the events of $\mathrm{Ml}$, stroke, or death (up to 5 years).

Results: In total, 734744 participants from the farmer cohort and 238311 from the control cohort aged between 40 and 70 were included. The age-adjusted incidence of MI was 0.766 and 0.585 per 1000 person-years in the farmer and control cohorts, respectively. That of stroke was 0.559 and 0.321 per 1000 person-years in both cohorts, respectively. In farmers, the risk factors for MI included male sex, age, personal history of hypertension, diabetes, current smoking, creatinine, metabolic syndrome components (blood pressure, triglycerides, and high-density lipoprotein cholesterol). Those for stroke included male sex, age, personal history of hypertension, diabetes, current smoking, high $\gamma$-glutamyl transferase, and metabolic syndrome components (blood pressure, triglycerides, and high-density lipoprotein cholesterol). The prediction model showed an area under the receiver operating characteristic curve of 0.735 and 0.760 for $\mathrm{Ml}$ and stroke, respectively, in the farmer cohort.

Conclusions: Farmers had a higher age-adjusted incidence of $\mathrm{Ml}$ and stroke. They also showed distinct patterns in cardiovascular risk factors compared to the general population.

Key words: Myocardial infarction, Stroke, Cardiovascular diseases, Coronary artery diseases, Cerebrovascular diseases, Farmers

\section{INTRODUCTION}

Received: April 24, 2020 Accepted: May 29, 2020

Corresponding author: Sang Baek Koh, MD, PhD

Department of Preventive Medicine, Yonsei University Wonju College of Medicine, 20 Ilsan-ro, Wonju 26426, Korea

E-mail: kohhj@yonsei.ac.kr

This is an Open Access article distributed under the terms of the Creative Commons Attribution Non-Commercial License (https://creativecommons.org/licenses/by$\mathrm{nc} / 4.0 / /)$ which permits unrestricted non-commercial use, distribution, and reproduction in any medium, provided the original work is properly cited.
Farmers in Korea face high levels of morbidity due to the declining farming population, aging workforce, and high labor intensity $[1,2]$. Compared to the general population, farmers have a greater risk of not only musculoskeletal diseases related to injury $[3,4]$, but also cardiovascular diseases (CVD) $[5,6]$, respiratory diseases $[7,8]$, and skin diseases $[9,10]$. Moreover, limited access to healthcare in rural areas is also one of the main causes of negative health outcomes in this population 


\section{[11,12].}

Myocardial infarction (MI) and stroke are among the major causes of death worldwide $[13,14]$. Due to advances in medical treatments, the rate of complications and death following $\mathrm{Ml}$ and stroke has substantially declined. However, these events typically cause a heavy medical burden, as they are often accompanied by chronic complications. Therefore, preventative approaches for early interventions by identifying high-risk groups among the population are very important. On the basis of identified risk factors, prediction models of $\mathrm{Ml}$ and stroke events among the general population have been developed $[15,16]$.

However, farmers receive chronic occupational exposure to physical and chemical factors distinct from those experienced by the general population [17-19]. Thus, patterns of disease in farmers may differ from those in the general population. Nonetheless, research on the health of farmers is limited to smallscale studies, and there is a lack of research on the incidence, risk factors, and prediction models for CVDs in this population using large-scale medical insurance billing information. This study drew data from the National Health Insurance Service of Korea (NHIS) database, analyzed the incidence and risk factors of $\mathrm{Ml}$ and stroke among farmers compared to the general population, developed a prediction model for the risk of disease in the next 5 years, and validated the prediction model.

\section{METHODS}

\section{Data Source and Study Approval}

This nationwide population-based cohort study was conducted using the NHIS database [20]. Korea has a single-payer insurance system that covers $99 \%$ of the entire population. The NHIS database includes enrollees' socioeconomic information, healthcare usage information, and diagnosis and treatment information. Information on regular health examinations and lifestyle patterns was extracted from the general health examination database. General health examinations are conducted once every 2 years for all employees, heads of households, and individuals aged 40 and above.

\section{Study Population}

The farmer cohort was established based on a customized database of farming households (Figure 1). Farming households were selected among farmers and fishers who were local policyholders of NHIS and who were eligible for reduced insurance contribution premiums on the basis of living in agricultural or fishing communities. The inclusion criteria were as follows: (1) those aged 40 to 89 in late 2010, (2) those who received a general health examination at least once during the indexing period from 2010 and 2011, and (3) those with no history or diagnosis of Ml or stroke before the general health examination during the index period. Those who reported a history of these diseases in the survey given as a part of the general health examination or those who used related healthcare services were excluded from the analysis. The index date of each individual was the date of the general health examination during the index period (Figure 2).

The comparison cohort was the National Health Insurance Service-National Sample Cohort (NHIS-NSC) [21]. This sample cohort is a $2 \%$ stratified random sample of the entire population based on sex, age, insurance type, insurance premium quartile, and region. The available data include health service use and the results of general health examinations from 2002 to 2015. Due to data availability, the age in the comparison cohort was calculated based on age figures in late 2009, and the index period ran from 2009 to 2010. In the comparison cohort, similarly to the farmer cohort, those who were aged 40 to 89 , those who received general health examinations at least once during the index period, and those without a history of $\mathrm{Ml}$ and stoke were included in the analytic sample. Individuals in these 2 cohorts were followed from the index date until the event of Ml, stroke, or their death (up to 5 years).

\section{Diagnosis of Incident Myocardial Infarction and Stroke}

For the diagnosis of MI and stroke, the International Classification of Diseases, 10th revision (ICD-10) operational definitions, which consider imaging results, procedures, and medication prescriptions for examination and treatment, were employed.

An ICD-10 code of I21 (acute transmural MI) was used for the diagnosis of MI. The diagnosis was limited to cases for which the patient was hospitalized at a medical facility and received both tests for biomarkers (troponin T, troponin I, or creatinine kinase-MB) and percutaneous coronary intervention (coronary angiography, percutaneous transluminal coronary angioplasty, percutaneous coronary stent placement, percutaneous coronary atherectomy, percutaneous coronary thrombectomy, aortocoronary bypass graft, or off-pump coronary artery bypass surgery) [22]. 


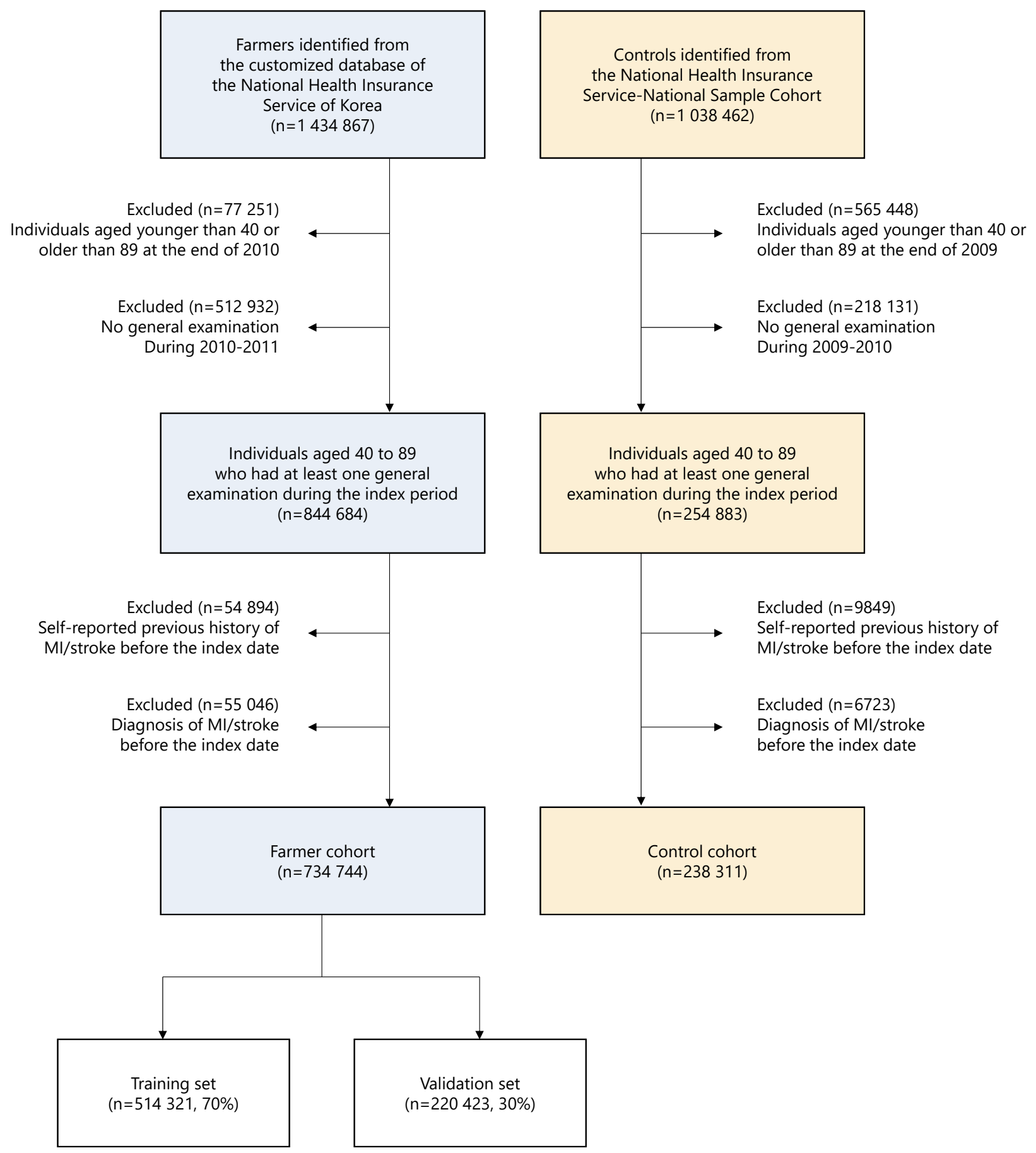

Figure 1. Participant selection flow chart. The National Health Insurance Service was our data source. A total of 734744 farmers and 238311 controls were recruited from the customized database and the National Sample Cohort. Of the farmers, 514321 (70\%) were used for training of the prediction model and 220423 (30\%) were used for validation. MI, myocardial infarction.

Stroke was diagnosed based on ICD-10 codes of 160 (nontraumatic subarachnoid hemorrhage), 161 (non-traumatic intracerebral hemorrhage), 162 (other and unspecified non-traumatic intracranial hemorrhage), 163 (cerebral infarction), or 164 (stroke, not specified as hemorrhage or infarction). This diagnosis was assigned in cases where the patient was hospitalized in a medical facility, underwent the necessary imaging exams for diagnosis (magnetic resonance imaging, computed 


\section{Farmers}

The customized database of the National Health Insurance Service of Korea

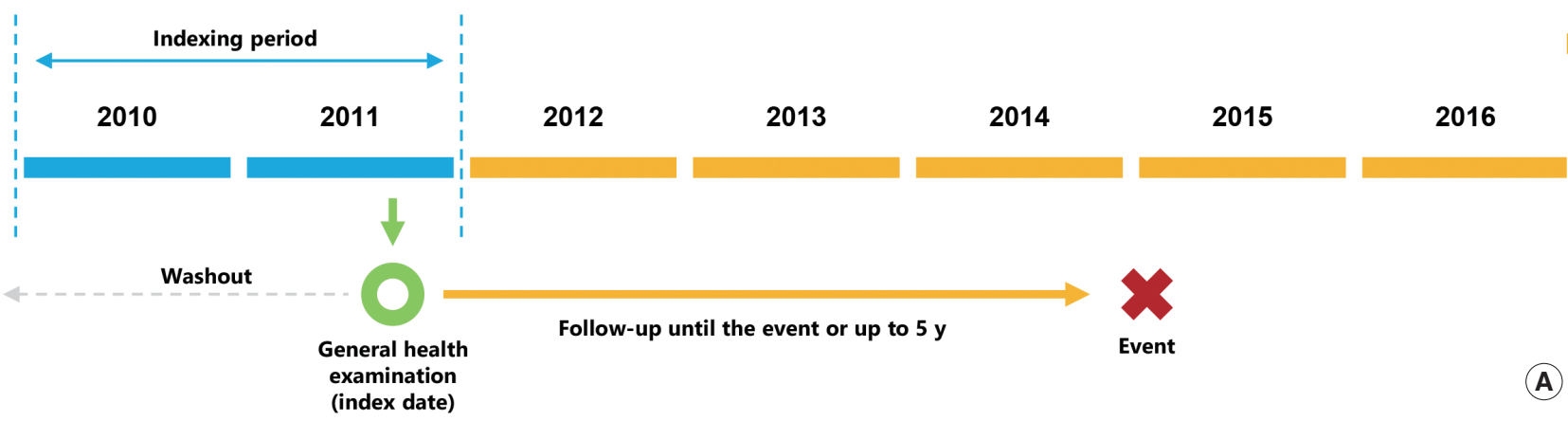

Controls

The National Health Insurance Service-National Sample Cohort

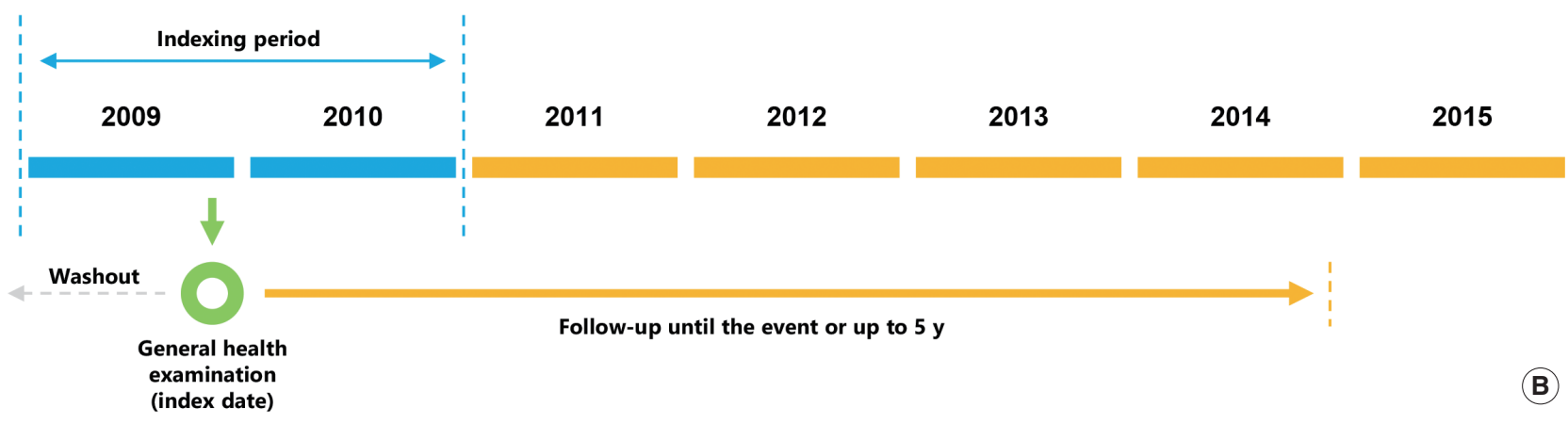

Figure 2. Schematic illustration of study design. Because of data availability, farmers (A) and controls (B) were recruited between 2010-2011 and 2009-2010, respectively. All participants were followed up from the index date (the day when a participant had a general health examination) until an event of interest or 5 years. Any participants with a previous history or diagnosis of myocardial infarction and/or stroke were excluded from the study.

tomography, or angiography for the cranial area), and received a percutaneous intervention (percutaneous thrombectomy or thrombolysis) or was prescribed related medication (aspirin, cilostazol, clopidogrel, ticlopidine, triflusal, heparin, bemiparin, reviparin, enoxaparin, dalteparin, fondaparinux, nadroparin, parnaparin, rivaroxaban, dabigatran, apixaban, coumadin, sarpogrelate, indobufen, mesoglycan, dipyridamole, or tissue plasminogen activator). Unlike patients with $\mathrm{Ml}$, stroke patients receive a percutaneous intervention only when they arrive within a specified time period (the "golden time") after the stroke occurs. Since percutaneous interventions are therefore only given to a limited number of patients, in order to identify more patients, an operational definition that includes the prescription of medications as conservative treatments is commonly used [23].

\section{Definition of Metabolic Syndrome}

According to the criteria of the National Cholesterol Education Program Adult Treatment Panel, metabolic syndrome was defined as the presence of at least 3 of the following 5 factors [24]: (1) waist circumference more than $90 \mathrm{~cm}$ in male and $80 \mathrm{~cm}$ in female, (2) blood triglyceride levels more than $150 \mathrm{mg} / \mathrm{dL}$ or the use of medication for hyperlipidemia, (3) serum high-density lipoprotein cholesterol below $40 \mathrm{mg} / \mathrm{dL}$ for male and below $50 \mathrm{mg} / \mathrm{dL}$ for female, (4) blood pressure more than 130/85 $\mathrm{mmHg}$ or the use of medication for hypertension, and (5) fasting blood sugar levels over $100 \mathrm{mg} / \mathrm{dL}$ or the use of medication for diabetes.

\section{Statistical Analysis}

In each cohort, for the incidence rate of $\mathrm{MI}$ and stroke, estimates for each group, as well as the age-standardized esti- 
mates based on the standard age structure in Korea in 2010, were calculated. In order to investigate the independent risk factors of $\mathrm{Ml}$ and stroke in each cohort, Cox proportional hazards regression using time-to-outcome variables from the start of the follow-up period was conducted. In selecting the variables, the proportional hazards assumption and multicollinearity were tested.

In order to construct the 5-year prediction model for MI and stroke, multivariate logistic regression was used. For the training of the model to fit the data, data from $70 \%$ of the participants in the farmer cohort were used. The data from the remaining $30 \%$ of the participants in the farmer cohort were used to test the accuracy of the developed model. The main measure to evaluate the performance of the prediction model was the area under receiver operating characteristic curve (AUROC).

All statistical analyses were conducted using SAS version 9.4 (SAS Institute Inc., Cary, NC, USA). Statistical significance was defined using a threshold of below $5 \%$.

\section{Data and Code Availability}

The NHIS provided the data that support the methods and results of this study. Access to the data is possible under the data use agreement for this study in a limited manner and cannot be given publicly. More detailed information about the data can be obtained on the following website: https://nhiss. nhis.or.kr/bd/ab/bdaba013eng.do. All statistical code used in the study is available in the following public repository: http:// dx.doi.org/10.17632/frwm44m3vz.1.

\section{Ethics Statement}

All data were anonymized to protect individual privacy. This study was approved by the Korean National Institute for Bioethics Policy (NHIS-2018-1-308), and since the study used anonymous data, it was not necessary to receive informed consent from each participant.

\section{RESULTS}

\section{Study Population}

The farmer cohort comprised 734744 individuals in the customized NHIS database, and the comparison cohort comprised 238311 individuals (Figure 1). The demographic characteristics and the general health examination results of these individuals at the index time are summarized in Table 1. The farmers
Table 1. Baseline characteristics by cohort

\begin{tabular}{|c|c|c|}
\hline Characteristics & $\begin{array}{c}\text { Farmer } \\
(\mathrm{n}=734744)\end{array}$ & $\begin{array}{c}\text { Control } \\
(n=238311)\end{array}$ \\
\hline Age (y) & $62.82 \pm 10.36$ & $54.31 \pm 10.16$ \\
\hline \multicolumn{3}{|l|}{ Sex } \\
\hline Male & $559521(76.1)$ & $113230(47.5)$ \\
\hline Female & $175223(23.8)$ & $125066(52.5)$ \\
\hline Body mass index (kg/m²) & $23.92 \pm 3.07$ & $24.00 \pm 3.04$ \\
\hline Waist circumference (cm) & $83.45 \pm 8.61$ & $81.13 \pm 8.61$ \\
\hline \multicolumn{3}{|l|}{ Self-reported lifestyle } \\
\hline Alcohol intake & $323869(44.1)$ & $96720(40.6)$ \\
\hline \multicolumn{3}{|l|}{ Smoking } \\
\hline Ever & 331767 (75.5) & $83362(35.0)$ \\
\hline Current & $182220(24.8)$ & 45989 (19.3) \\
\hline \multicolumn{3}{|l|}{ Self-reported history } \\
\hline Hypertension & 211239 (38.76) & 49717 (31.25) \\
\hline Diabetes mellitus & 75028 (13.9) & $16928(10.7)$ \\
\hline Dyslipidemia & $19683(3.6)$ & $8548(5.4)$ \\
\hline Systolic blood pressure (mmHg) & $127.4 \pm 15.7$ & $124.2 \pm 15.5$ \\
\hline Diastolic blood pressure $(\mathrm{mmHg})$ & $78.68 \pm 9.80$ & $77.13 \pm 10.18$ \\
\hline Creatinine (mg/dL) & $1.00 \pm 1.02$ & $1.01 \pm 1.10$ \\
\hline$\gamma$-glutamyl transferase (U/L) & $47.68 \pm 73.19$ & $37.42 \pm 51.89$ \\
\hline Fasting glucose (mg/dL) & $103.20 \pm 27.73$ & $100.20 \pm 25.40$ \\
\hline Total cholesterol (mg/dL) & $196.10 \pm 42.59$ & $200.20 \pm 42.29$ \\
\hline Triglyceride (mg/dL) & $152.7 \pm 129.7$ & $140.9 \pm 115.5$ \\
\hline High-density lipoprotein (mg/dL) & $53.78 \pm 30.36$ & $55.90 \pm 31.04$ \\
\hline Low-density lipoprotein (mg/dL) & $114.80 \pm 66.00$ & $11.98 \pm 78.07$ \\
\hline Metabolic equivalent of task/wk & $550.3 \pm 683.5$ & $656.7 \pm 706.0$ \\
\hline \multicolumn{3}{|l|}{ Metabolic syndrome } \\
\hline Yes & $238025(32.4)$ & $64509(27.1)$ \\
\hline \multicolumn{3}{|l|}{ Component } \\
\hline Waist circumference & $197541(26.9)$ & $53976(22.7)$ \\
\hline Triglyceride & $276179(37.6)$ & 79074 (33.2) \\
\hline High-density lipoprotein & $187839(25.6)$ & $67623(28.4)$ \\
\hline Glucose & 321504 (43.8) & $88230(37.0)$ \\
\hline Blood pressure & 439685 (59.8) & 117545 (49.3) \\
\hline \multicolumn{3}{|l|}{ No. of components } \\
\hline 0 & 101162 (13.8) & $49396(20.7)$ \\
\hline 1 & $193090(26.3)$ & $65657(27.5)$ \\
\hline 2 & 202467 (27.6) & $58734(24.6)$ \\
\hline 3 & 146558 (19.9) & 39972 (16.8) \\
\hline 4 & $72285(9.8)$ & $19278(8.1)$ \\
\hline 5 & $19182(2.6)$ & $5259(2.2)$ \\
\hline
\end{tabular}

Values are presented as number (\%) or mean \pm standard deviation.

were statistically significantly older than individuals in the comparison cohort ( $62.82 \pm 10.36$ vs. $54.31 \pm 10.16$ years; $p<0.001$ ). The proportion of males was significantly higher among farm- 
ers than among the general population (76.1 vs. $47.5 \%$; $p<$ 0.001 ). A history of various chronic diseases, including metabolic syndrome, was more prevalent in farmers than in the general population.

\section{Incidence of Myocardial Infarction and Stroke}

The 734774 individuals in the farmer cohort and the 238311 individuals in the comparison cohort were followed longitudinally from the index time for up to 5 years.

In the farmer cohort, 3860 incident Mls were recorded over 3662916.9 person-years of follow-up (group incidence rate, $1.053 / 1000$ person-years). In the comparison cohort, 630 incident Mls were recorded over 11898313.5 person-years of follow-up (group incidence rate, $0.529 / 1000$ person-years). The age-standardized incidence rates based on the 2010 standard population structure were $0.766 / 1000$ person-years in the farmer cohort and $0.585 / 1000$ person-years in the comparison cohort.

In the farmer cohort, 3162 incident strokes were recorded over 3665033.8 person-years of follow-up (group incidence rate, $0.863 / 1000$ person-years). In the comparison cohort, 322 incident strokes were recorded over 1190694.9 person-years (group incidence rate, $0.271 / 1000$ person-years). The age- standardized incidence rates based on the 2010 standard population structure were $0.559 / 1000$ person-years in the farmer cohort and $0.321 / 1000$ person-years in the comparison cohort.

\section{Risk Factors for Incident Disease}

The independent risk factors of $\mathrm{Ml}$ and stroke in the farmer cohort and the comparison cohort are summarized in Table 2 .

The common risk factors of $\mathrm{Ml}$ across the two cohorts were male sex, old age, current smoking, a history of diabetes, low serum $\gamma$-glutamyl transferase levels, hypertension, high serum triglyceride levels, and low serum high-density lipoprotein cholesterol levels. A history of hypertension was statistically significant in the farmer cohort (hazard ratio [HR], 1.217; 95\% confidence interval [CI], 1.114 to 1.329 ) and not in the comparison cohort (HR, $0.958 ; 95 \% \mathrm{Cl}, 0.771$ to 1.191$)$, but the HRs in each cohort were not significantly different.

The common risk factors of stroke in the two cohorts were old age, current smoking, and a history of diabetes. In the farmer cohort, male sex was associated with a slightly higher risk of stroke (HR, 1.295; $95 \% \mathrm{Cl}, 1.157$ to 1.451 ), but in the comparison cohort, no effect of sex was observed (HR, $0.941 ; 95 \% \mathrm{Cl}, 0.663$ to 1.335$)$. Similarly, a self-reported history and a presence of

Table 2. Risk factors for incident myocardial infarction (MI) in the farmer and control cohorts

\begin{tabular}{|c|c|c|c|c|}
\hline \multirow{2}{*}{ Characteristics } & \multicolumn{2}{|c|}{ MI } & \multicolumn{2}{|c|}{ Stroke } \\
\hline & Farmer & Control & Farmer & Control \\
\hline Sex, male & $2.262(2.012,2.538)$ & $2.475(1.894,3.236)$ & $1.295(1.157,1.451)$ & $0.941(0.663,1.335)$ \\
\hline Age (y) & $1.053(1.048,1.057)$ & $1.059(1.049,1.069)$ & $1.092(1.087,1.098)$ & $1.091(1.077,1.105)$ \\
\hline \multicolumn{5}{|l|}{ Personal history } \\
\hline Hypertension & $1.217(1.114,1.329)$ & $0.958(0.771,1.191)$ & $1.119(1.016,1.232)$ & $1.085(0.797,1.479)$ \\
\hline Diabetes mellitus & $1.699(1.540,1.875)$ & $1.819(1.420,2.329)$ & $1.538(1.374,1.722)$ & $1.761(1.254,2.473)$ \\
\hline Dyslipidemia & $0.752(0.615,0.918)$ & $0.749(0.507,1.106)$ & $0.672(0.515,0.876)$ & $0.664(0.353,1.250)$ \\
\hline \multicolumn{5}{|l|}{ Smoking } \\
\hline Ever & $0.968(0.875,1.071)$ & $1.267(0.954,1.684)$ & $1.036(0.924,1.160)$ & $1.356(0.882,2.084)$ \\
\hline Current & $1.833(1.655,2.030)$ & $2.042(1.591,2.622)$ & $1.604(1.426,1.805)$ & $2.033(1.369,3.020)$ \\
\hline Body mass index $\left(\mathrm{kg} / \mathrm{m}^{2}\right)$ & $0.993(0.978,1.008)$ & $1.010(0.972,1.050)$ & $0.977(0.960,0.993)$ & $0.982(0.932,1.034)$ \\
\hline Creatinine (mg/dL) & $1.031(1.014,1.048)$ & $1.026(0.986,1.069)$ & $1.010(0.977,1.045)$ & $1.042(1.007,1.077)$ \\
\hline$\gamma$-glutamyl transferase (U/L) & $0.998(0.997,0.999)$ & $0.997(0.995,1.000)$ & $1.001(1.001,1.002)$ & $1.001(0.999,1.003)$ \\
\hline \multicolumn{5}{|l|}{ Metabolic syndrome components } \\
\hline High blood pressure & $1.292(1.166,1.431)$ & $1.611(1.260,2.060)$ & $1.393(1.240,1.565)$ & $1.315(0.923,1.875)$ \\
\hline High waist circumference & $1.070(0.971,1.179)$ & $1.150(0.905,1.461)$ & $1.063(0.953,1.186)$ & $1.134(0.812,1.583)$ \\
\hline High glucose & $1.014(0.932,1.103)$ & $0.955(0.771,1.183)$ & $1.003(0.914,1.100)$ & $1.055(0.783,1.422)$ \\
\hline High triglycerides & $1.354(1.253,1.464)$ & $1.644(1.349,2.003)$ & $1.127(1.033,1.230)$ & $1.027(0.779,1.354)$ \\
\hline Low high-density lipoprotein & $1.309(1.204,1.422)$ & $1.377(1.122,1.689)$ & $1.132(1.029,1.244)$ & $1.156(0.870,1.537)$ \\
\hline
\end{tabular}

Values are presented as hazard ratio (95\% confidence interval). 

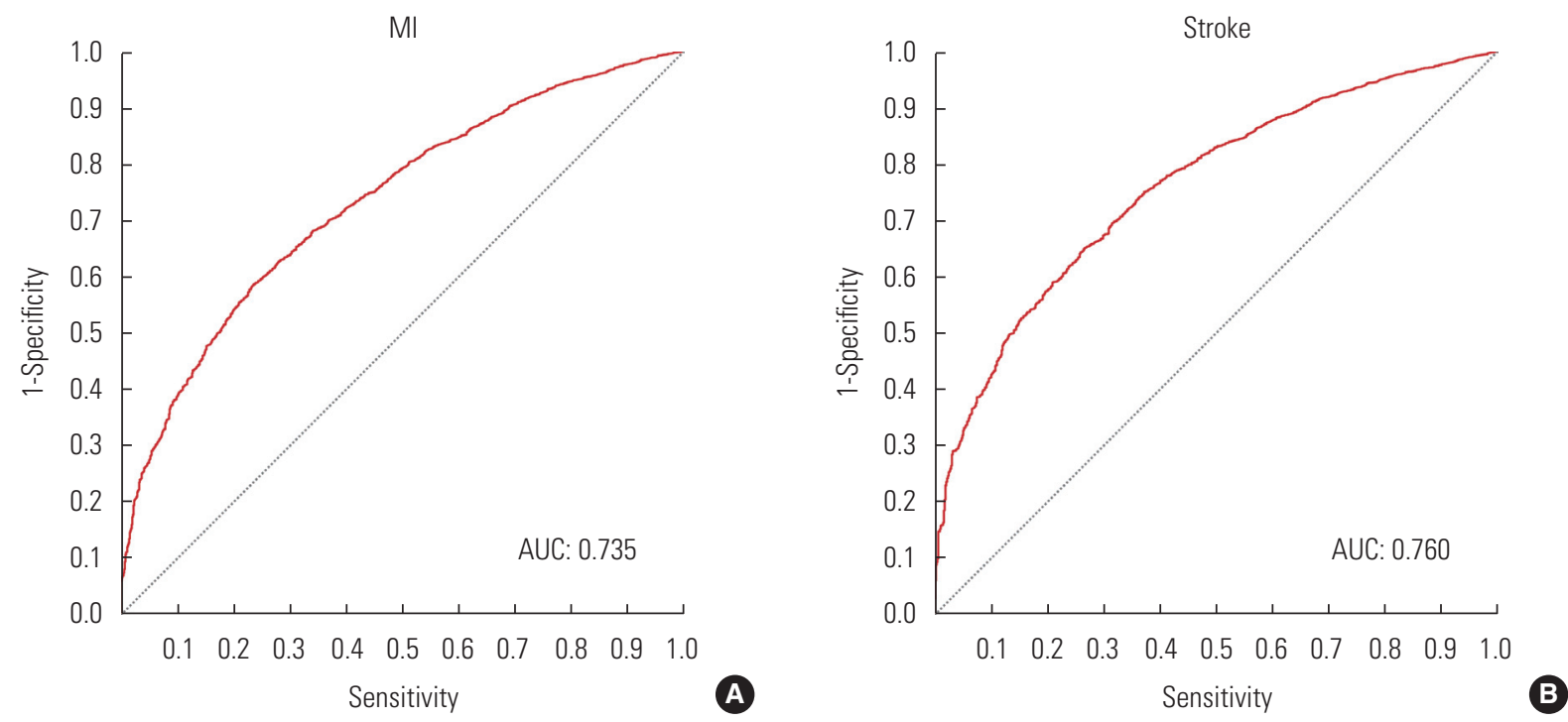

Figure 3. Receiver operating characteristics curve. Five-year prediction model for incident MI (A) and stroke (B). MI, myocardial infarction; AUC, area under the curve.

hypertension were statistically significant risk factors in the farmer cohort (HR, 1.119, 95\% Cl, 1.016 to 1.232 and HR, 1.393; $95 \% \mathrm{Cl}, 1.240$ to 1.565 , respectively), but not in the comparison cohort (HR, 1.085; $95 \% \mathrm{Cl}, 0.797$ to 1.479 and $\mathrm{HR}, 1.315$; $95 \% \mathrm{Cl}, 0.923$ to 1.875 , respectively). However, the HRs were not significantly different. High serum $\gamma$-glutamyl transferase levels were identified as a risk factor of stroke in the farmer cohort (HR, 1.001; 95\% Cl, 1.001 to 1.002), but were not significant in the comparison cohort (HR, 1.001; $95 \% \mathrm{Cl}, 0.999$ to 1.003). Although the HRs were not significantly different, high serum triglyceride levels ( $\mathrm{HR}, 1.127 ; 95 \% \mathrm{Cl}, 1.033$ to 1.230$)$ and low serum high-density lipoprotein levels (HR, 1.132; 95\% $\mathrm{Cl}, 1.024$ to 1.244 ) were identified as significant risk factors in the farmer cohort, but were not statistically significant in the comparison cohort (HR, 1.027; 0.779 to 1.354 and HR, 1.156; $95 \% \mathrm{Cl}, 0.870$ to 1.537 , respectively).

\section{Five-year Prediction Model in the Farmer}

\section{Population}

In order to predict $\mathrm{MI}$ and stroke within 5 years from the date of the general health examination in the farmer cohort, a prediction model for each disease was developed, using 70\% $(n=514321)$ of the data from the farmer cohort $(n=734744)$ for the development and training of the models, and the remaining 30\% ( $n=220423$ ) as a validation set. The accuracy of the 5 -year prediction models for $\mathrm{Ml}$ and stroke are presented in Figure 3.

\section{DISCUSSION}

This study found that the group incidence rate and age-standardized mortality rate of $\mathrm{Ml}$ and stroke were substantially higher in the farmer cohort than in the general population. Moreover, the risk factors of these 2 diseases in farmers differed from the known risk factors in the general population.

Previous studies reported that the incidence and prevalence of various health conditions, including musculoskeletal, CVD, and respiratory diseases, were higher in farmers $[4-6,8,10]$. Factors that contribute to the higher health risk in farmers may include (1) chemical factors such as exposure to gases or harmful chemical substances in pesticides and agricultural machinery; (2) physical factors such as long working hours, high-intensity labor, ergonomic burden, high temperatures, and noise pollution; and (3) psychological factors such as agricultural stress [9,25-29]. Therefore, health examinations and provision of health services for the diagnosis and prediction of diseases in farmers, who are a high-risk population, are imperative. However, health access is actually substantially lower in the farmer population than in other populations, and specialized health examinations and monitoring of major diseases for farmers are limited to the management of some musculoskeletal diseases [11,12].

Compared to the general population, farmers demonstrated a different pattern of risk factors for $\mathrm{Ml}$ and stroke. One of the main differences was that sex had no significant effect on stroke 
risk in the general population, but the risk was higher in males than in females in the farmer population. The role of sex in stroke risk has been the topic of ongoing discussions [30,31]. It is known that in subtypes of stroke other than subarachnoid hemorrhage there are no differences according to sex [32]. Higher exposure to occupational risk factors in male farmers than in female farmers might have contributed to the difference in risk associated with sex observed in the 2 cohorts. However, since the data analyzed in this study did not include information on individuals' intensity and duration of labor or exposure to occupational risk factors, potential mechanisms and explanations can only be suggested to a limited degree.

A history of hypertension was an independent risk factor for $\mathrm{Ml}$ and stroke among farmers, but not among the general population. Since a higher proportion of farmers were elderly compared to the general population, hypertension may have had a longer disease period in the farmer cohort, contributing to the identification of history of hypertension as a risk factor. It is known that factors such as use of pesticides, long working hours, physical exertion, and agricultural stress cause hemodynamic changes, including hypertension [33-36]. Moreover, lifestyle choices that are more prevalent in farmers than in people in other occupational categories, such as drinking, smoking, and certain eating habits, are important risk factor of hypertension. Therefore, it is possible that hypertension is experienced starting at an earlier age in farmers than in the general population, and the interaction between pesticide exposure and hypertension may have caused the different contribution of a history of hypertension to disease incidence in the 2 cohorts.

The high occupational stress of farmers caused by poor working conditions, environmental and climate conditions, economic problems, uncertainty, and social isolation can influence hypertension. However, the HRs of the independent factors examined were not substantially different between the 2 cohorts, and considering the number of participants, the interpretation of statistical significance requires some caution. Moreover, the data analyzed lacked information on the age at onset of hypertension, length of disease, or exposure to pesticides, so the independent effects of each factor could not be examined in detail. High serum $\gamma$-glutamyl transferase levels were also found to significantly increase the risk of $\mathrm{Ml}$ in farmers, but not in the general population. Although it is difficult to propose an exact mechanism, elevated serum $\gamma$-glutamyl transferase levels due to the exposure to pesticides and other agricultural chemicals may contribute to this observed difference [37-39].
The AUROCs of the prediction models for MI or stroke from the time of general health examination up to 5 years among farmers were 0.735 and 0.760 , respectively. These figures suggest a moderate fit of the models; in other words, the models did not demonstrate a high predictive accuracy. This study and the prediction models did not use additional survey information on occupational factors that could improve the predictive accuracy of the models among farmers. Therefore, although these models were developed and validated using data from the farming population, there is a limitation to their predictive accuracy.

The main limitation of this study is that data specific to the farming population were not used as analytic variables when the risk factors of $\mathrm{Ml}$ and stroke were identified and prediction models were built. For example, if variables such as type of crops cultivated, occupational environment, main tasks, labor duration and intensity, length of work, types of pesticides used, amount of pesticides used, the environment where the pesticides were used, and whether appropriate protective gear was used had been analyzed, the risk factors and mechanisms in this population could have been examined more clearly. Another limitation of this study is that all analyses and predictions were based on the underlying health status and lifestyle factors at the time of the general health examination. Health status and lifestyle choices are not fixed in each individual, and in a recent large-scale study, it was reported that adjustments in lifestyle choices can alter the progression of metabolic syndrome and reduce the risk of disease [40].

There may also have been misclassification of the farmer cohort and the general population cohort. The farmer cohort was constructed by extracting households included in the administrative registration data. Therefore, family members who were not farmers could have been included in the farmer cohort. By limiting the study population to individuals aged 40 to 80 , we attempted to minimize the possibility of misclassification. Lastly, the NHIS-NSC cohort used to represent the general population was based on the entire population and therefore included a small number of farmers. This misclassification might have led to underestimation of the incidence rate of disease and independent risk factors in farmers compared to the general population.

Despite the above limitations, the strengths of this study are that it calculated the national age-standardized incidence rates of $\mathrm{Ml}$ and stroke among farmers compared to the general population, which has rarely been studied, and that it investigated the independent risk factors of each disease in the 
farmer cohort and the general population. Further research that incorporates more detailed survey items and a longer follow-up period is necessary.

\section{CONFLICT OF INTEREST}

The authors have no conflicts of interest associated with the material presented in this paper.

\section{FUNDING}

This work was carried out with the support of the Cooperative Research Program for Agriculture Science \& Technology Development (project No. PJ01250901) and the Rural Development Administration, Republic of Korea.

\section{ACKNOWLEDGEMENTS}

None.

\section{AUTHOR CONTRIBUTIONS}

Conceptualization: SL, SBK. Data curation: SL, HSK. Formal analysis: SL. Funding acquisition: SBK. Methodology: SL, HL, HSK, SBK. Project administration: SBK. Visualization: SL. Writing original draft: SL. Writing - review \& editing: SL, HL, HSK, SBK.

\section{ORCID}

Solam Lee https://orcid.org/0000-0001-6458-9449

Hunju Lee https://orcid.org/0000-0003-0089-0069

Hye Sim Kim https://orcid.org/0000-0002-7431-9722

Sang Baek Koh https://orcid.org/0000-0001-5609-6521

\section{REFERENCES}

1. Kim YO, Lee BK. Structural change of agricultural population and farming characteristics of women's management. J Rural Soc 2000;10:7-35 (Korean).

2. Lim HB. The change of rural population in the process of Korean rural planning. J Agric Educ Hum Resour Dev 2005;37(4): 199-223 (Korean).

3. Kee DH. Survey of musculoskeletal disorders for farmers. J Korean Soc Saf 2009;24(3):59-64 (Korean).

4. Kim KR, Lee KS, Kim HC, Song EY. Health status and musculo- skeletal workload of red pepper farmers. J Ergon Soc Korea 2009;28(3):7-15 (Korean).

5. Brackbill RM, Cameron LL, Behrens V. Prevalence of chronic diseases and impairments among US farmers, 1986-1990. Am J Epidemiol 1994;139(11):1055-1065.

6. Asgary R, Galson S, Shankar H, O'Brien C, Arole S. Hypertension, pre-hypertension, and associated risk factors in a subsistent farmer community in remote rural central India. J Public Health 2013;21(3):251-258.

7. Wunschel J, Poole JA. Occupational agriculture organic dust exposure and its relationship to asthma and airway inflammation in adults. J Asthma 2016;53(5):471-477.

8. Linaker C, Smedley J. Respiratory illness in agricultural workers. Occup Med (Lond) 2002;52(8):451-459.

9. Spiewak R. Pesticides as a cause of occupational skin diseases in farmers. Ann Agric Environ Med 2001;8(1):1-5.

10. Susitaival P. Occupational skin diseases in farmers and farm workers. In: Kanerva L, editor. Handbook of occupational dermatology. Berlin: Springer; 2000, p. 924-931.

11. Kim DJ. General and emergency medical use status in rural residents and implications. Health Welf Issue Focus 2012;159: 1-8 (Korean).

12. Yi YJ, Kim EJ. The effects of accessibility to medical facilities and public transportation on perceived health of urban and rural elderly: using generalized ordered logic model. J Korean Reg Dev Assoc 2015;27(1):65-88 (Korean).

13. Roth GA, Johnson C, Abajobir A, Abd-Allah F, Abera SF, Abyu G, et al. Global, regional, and national burden of cardiovascular diseases for 10 causes, 1990 to 2015. J Am Coll Cardiol 2017; 70(1):1-25.

14. Feigin VL, Roth GA, Naghavi M, Parmar P, Krishnamurthi R, Chugh $\mathrm{S}$, et al. Global burden of stroke and risk factors in 188 countries, during 1990-2013: a systematic analysis for the Global Burden of Disease Study 2013. Lancet Neurol 2016; 15(9):913-924.

15. Eagle KA, Lim MJ, Dabbous OH, Pieper KS, Goldberg RJ, Van de Werf $F$, et al. A validated prediction model for all forms of acute coronary syndrome: estimating the risk of 6-month postdischarge death in an international registry. JAMA 2004; 291(22):2727-2733.

16. Jee SH, Park JW, Lee SY, Nam BH, Ryu HG, Kim SY, et al. Stroke risk prediction model: a risk profile from the Korean study. Atherosclerosis 2008;197(1):318-325.

17. Damalas CA, Koutroubas SD. Farmers' exposure to pesticides: toxicity types and ways of prevention. Toxics 2016;4(1):1. 
18. Vogelzang PF, van der Gulden JW, Folgering H, Kolk JJ, Heederik D, Preller L, et al. Endotoxin exposure as a major determinant of lung function decline in pig farmers. Am J Respir Crit Care Med 1998;157(1):15-18.

19. Coble J, Hoppin JA, Engel L, Elci OC, Dosemeci M, Lynch CF, et al. Prevalence of exposure to solvents, metals, grain dust, and other hazards among farmers in the Agricultural Health Study. $J$ Expo Anal Environ Epidemiol 2002;12(6):418-426.

20. Seong SC, Kim YY, Khang YH, Park JH, Kang HJ, Lee H, et al. Data resource profile: the national health information database of the National Health Insurance Service in South Korea. Int J Epidemiol 2017;46(3):799-800.

21. Lee J, Lee JS, Park SH, Shin SA, Kim K. Cohort profile: the national health insurance service-national sample cohort (NHISNSC), South Korea. Int J Epidemiol 2017;46(2):e15.

22. Kimm H, Yun JE, Lee SH, Jang Y, Jee SH. Validity of the diagnosis of acute myocardial infarction in Korean national medical health insurance claims data: the Korean Heart Study (1). Korean Circ J 2012;42(1):10-15.

23. Kim JY, Lee KJ, Kang J, Kim BJ, Han MK, Kim SE, et al. Development of stroke identification algorithm for claims data using the multicenter stroke registry database. PLoS One 2020;15(2): e0228997.

24. Grundy SM, Cleeman JI, Merz CN, Brewer HB Jr, Clark LT, Hunninghake $\mathrm{DB}$, et al. Implications of recent clinical trials for the national cholesterol education program adult treatment panel III guidelines. J Am Coll Cardiol 2004;44(3):720-732.

25. Sekhotha MM, Monyeki KD, Sibuyi ME. Exposure to agrochemicals and cardiovascular disease: a review. Int J Environ Res Public Health 2016;13(2):229.

26. Quinteros E, Ribó A, Mejía R, López A, Belteton W, Comandari A, et al. Heavy metals and pesticide exposure from agricultural activities and former agrochemical factory in a Salvadoran rural community. Environ Sci Pollut Res Int 2017;24(2):1662-1676.

27. Raksanam B, Taneepanichskul S, Robson MG, Siriwong W. Health risk behaviors associated with agrochemical exposure among rice farmers in a rural community, Thailand: a community-based ethnography. Asia Pac J Public Health 2014;26(6):588-595.

28. Andreatta S. Agrochemical exposure and farmworker health in the Caribbean: a local/global perspective. Hum Organ 1998; 57(3):350-358.

29. Faria NM, Fassa AG, Meucci RD, Fiori NS, Miranda VI. Occupational exposure to pesticides, nicotine and minor psychiatric disorders among tobacco farmers in southern Brazil. Neurotoxicology 2014;45:347-354.

30. Appelros P, Stegmayr B, Terént A. Sex differences in stroke epidemiology: a systematic review. Stroke 2009;40(4):1082-1090.

31. Roquer J, Campello AR, Gomis M. Sex differences in first-ever acute stroke. Stroke 2003;34(7):1581-1585.

32. Reeves MJ, Bushnell CD, Howard G, Gargano JW, Duncan PW, Lynch G, et al. Sex differences in stroke: epidemiology, clinical presentation, medical care, and outcomes. Lancet Neurol 2008; 7(10):915-926.

33. Anand M, Gulati A, Gopal K, Gupta GS, Khanna RN, Ray PK, et al. Hypertension and myocarditis in rabbits exposed to hexachlorocyclohexane and endosulfan. Vet Hum Toxicol 1990;32(6): 521-523.

34. Gordon CJ, Padnos BK. Prolonged elevation in blood pressure in the unrestrained rat exposed to chlorpyrifos. Toxicology 2000;146(1):1-13.

35. Morton WE, Crawford ED, Maricle RA, Douglas DD, Freed VH. Hypertension in Oregon pesticide-formulating workers. J Occup Med 1975;17(3):182-185.

36. Sandifer SH, Keil JE, Finklea JF, Gadsden RH. Pesticide effects on occupationally exposed workers: a summary of four years observation of industry and farm volunteers in South Carolina. IMS Ind Med Surg 1972;41(5):9-12.

37. Lee DH, Steffes MW, Jacobs DR Jr. Can persistent organic pollutants explain the association between serum gamma-glutamyltransferase and type 2 diabetes? Diabetologia 2008; 51(3):402-407.

38. Ha MH, Lee DH, Jacobs DR. Association between serum concentrations of persistent organic pollutants and self-reported cardiovascular disease prevalence: results from the National Health and Nutrition Examination Survey, 1999-2002. Environ Health Perspect 2007;115(8):1204-1209.

39. Lee $D H$, Gross MD, Steffes MW, Jacobs DR Jr. Is serum gammaglutamyltransferase a biomarker of xenobiotics, which are conjugated by glutathione? Arterioscler Thromb Vasc Biol 2008;28(4):e26-e28.

40. Park S, Lee S, Kim Y, Lee Y, Kang MW, Han K, et al. Altered risk for cardiovascular events with changes in the metabolic syndrome status: a nationwide population-based study of approximately 10 million persons. Ann Intern Med 2019;171(12): 875-884. 